\section{Oral histiocytic sarcoma in a cat}

\section{Slavomíra Néčová ${ }^{1}$, Stephen Cahalan ${ }^{2}$, Susan North ${ }^{3}$, Smita Das ${ }^{4}$}

1 Southfields Veterinary Specialists, Laindon, United Kingdom 2 Bridge Pathology Ltd. , Bristol, United Kingdom

3 Southfields Veterinary Specialists, Laindon, United Kingdom

4 Davies Veterinary Specialists, Higham Gobion, United Kingdom

\section{OBJECTIVES}

Feline histiocytic disease is poorly reported in the veterinary literature. Here we describe a tumour of the feline soft palate most consistent with histiocytic sarcoma. To the author's knowledge this is the first report of a feline histiocytic sarcoma of the oral cavity.

\section{METHODS}

A 15 year-old male neutered domestic shorthair cat presented with a mass on the caudal soft palate. An incisional biopsy was performed and sarcoma was highly suspected. Computed tomography (CT) of the patient's head and thorax identified a discrete, left-sided, soft tissue mass lesion cranial to the tonsil. Surgical removal achieved cytoreduction. Adjuvant lomustine chemotherapy was initiated but was discontinued after one treatment cycle due to pancreatitis.

\section{RESULTS}

Histopathology of the mass showed proliferation of large, polygonal to occasionally spindled neoplastic cells forming solid sheets and poorly defined streams. There was marked anisocytosis, anisokaryosis and frequent multinucleated giant cells. Periodic acid-Schiff and Ziehl-Neelsen stains were negative. Immunohistochemistry showed strong membranous HLA-DR positivity along with membranous CD18 immunopositivity within tumour macrophages. There was weaker CD18 and IBA 1 staining of neoplastic cells. Immunohistochemistry for Melan A, PNL2 and CD117 was negative. The patient was diagnosed with suspected local recurrence 3 months post-operatively and was euthanised 6 months after initial diagnosis due to disease progression.

\section{STATEMENT (CONCLUSIONS)}

Histiocytic sarcoma should be considered as a differential diagnosis in feline patients with an oral mass, especially if histopathology suggests a pleomorphic and poorly differentiated sarcoma. Immunohistochemistry for the confirmation of cell line origin would be strongly recommended.

\section{Unusual presentation of a bone metastasis in canine prostatic carcinoma}

\section{Slavomíra Néčová ${ }^{1}$, Poppy Winter ${ }^{2}$, Sarah Mason ${ }^{1}$}

1 Southfields Veterinary Specialists, Laindon, United Kingdom

2 Maltman Cosham Veterinary Clinic, Horsham, United Kingdom

\section{OBJECTIVES}

The objective of this case report is to describe the radiological appearance of an elbow metastasis from a prostatic carcinoma.

\section{METHODS}

An eight year old male neutered Chesapeake Bay Retriever presented with a few day history of dysuria and tenesmus. The dog was diagnosed with prostatic adenocarcinoma with urinary bladder involvement and regional lymph node metastases. The dog developed mild lameness on left fore limb one month after initial presentation which progressed over two weeks to non-weight bearing despite pain relief and anti-inflammatory medication. Significant left elbow thickening was present. The computed tomography (CT), arthrocentesis and FNA of macroscopically abnormal tissue was performed.

\section{RESULTS}

CT revealed a polyostic aggressive lesion, characterised by irregular periosteal proliferation, affecting the proximal ulna and radius, and humeral condyles. Mild cortical thinning and lysis at the anconeal and lateral coronoid processes was present. The primary differential for these changes was an inflammatory, degenerative and/or infectious process. Neoplasia was considered less likely due to the distribution of the lesions. Cell morphology of a changed bone was consistent with metastatic carcinoma. There were rare atypical cells within the joint fluid which also raised concern for a neoplastic process associated with the joint. Histopathological evaluation of the left elbow confirmed metastatic carcinoma with periosteal new bone formation, cartilage erosion and subchondral fibrosis.

\section{STATEMENT (CONCLUSIONS)}

This case demonstrates that metastatic carcinoma can radiologically appear as an inflammatory/infectious process and involve multiple bones including joint. Cytological or histological confirmation of similar changes in carcinoma patients is advised 